\title{
QUIVERS, QUASI-QUANTUM GROUPS AND FINITE TENSOR CATEGORIES
}

\author{
HUA-LIN HUANG, GONGXIANG LIU, AND YU YE
}

\begin{abstract}
We study finite quasi-quantum groups in their quiver setting developed recently by the first author. We obtain a classification of finite-dimensional pointed Majid algebras of finite corepresentation type, or equivalently a classification of elementary quasi-Hopf algebras of finite representation type, over the field of complex numbers. By the Tannaka-Krein duality principle, this provides a classification of the finite tensor categories in which every simple object has Frobenius-Perron dimension 1 and there are finitely many indecomposable objects up to isomorphism. Some interesting information of these finite tensor categories is given by making use of the quiver representation theory.
\end{abstract}

Keywords quasi-quantum group, tensor category, Hopf quiver 2000 MR Subject Classification 16W35, 18D20, 16G20, 16G60

\section{INTRODUCTION}

This paper is devoted to the classification of finite quasi-quantum groups and the associated representation theory, whence the classification of finite tensor categories [18], within the quiver setting developed recently in [28, 29]. The notion of quasi-Hopf algebras was introduced by Drinfeld [13] in connection with the Knizhnik-Zamolodchikov system of equations from conformal field theory. The definition of quasi-Hopf algebras is not selfdual, so there is a dual notion which is called Majid algebra after Shnider-Sternberg [37]. In accordance with Drinfeld's philosophy of quantum groups [12], we understand both of these mutually dual algebraic structures in the framework of quasi-quantum groups.

We focus on finite-dimensional pointed Majid algebras, or equivalently elementary quasi-Hopf algebras. Within this restriction, we can take full advantage of the quiver techniques to tackle the problems of classification and representation theory. Recall that by pointed it is meant that the simple subcoalgebras of the underlying coalgebras are one-dimensional. Dually, by elementary it is meant that the underlying algebras are finite-dimensional and their simple modules are one-dimensional. 
Quivers are oriented diagrams consisting of vertices and arrows. Due to Gabriel [19, 20], in the early 1970's quivers and their representations became widespread first in the representation theory of associative algebras. Nowadays these notions show up in various areas of mathematics and physics. What we rely on is still their combinatorial behavior and hence very handy applications in the study of algebraic structures and representation theory. In connection with Hopf algebras and quantum groups, Hopf quivers [11] and covering quivers [26] were introduced, see also [9, 10, 25, 6, 38] for related works. For the quiver setting of the broader class of quasi-quantum groups, it turns out that there is nothing new other than the Hopf quivers. In principle, it is shown in $[28,29]$ that pointed Majid algebras and elementary quasi-Hopf algebras can be constructed on Hopf quivers exhaustively with a help of the projective representation theory of groups and a proper deformation theory.

According to the well-known Tannaka-Krein duality principle (see for instance $[35,5]$ ), finite quasi-quantum groups are deeply related to finite tensor categories. More precisely, the representation categories of finitedimensional quasi-Hopf algebras and the corepresentation categories of finitedimensional Majid algebras are finite tensor categories; conversely, finite tensor categories with some mild conditions are obtained in this way.

In recent years finite tensor categories and finite quasi-quantum groups have been intensively studied by Etingof, Gelaki, Nikshych, Ostrik, and many other authors. In [17], the fusion categories, that is, the semisimple finite tensor categories, are investigated in depth and a number of general properties are obtained. A systematic study of not necessarily semisimple finite tensor categories initiated in [18], and some classification results were obtained in $[14,15,22,16]$ through concrete constructions of elementary quasi-Hopf algebras.

The aim of this paper is to classify finite-dimensional pointed Majid algebras of finite corepresentation type, or equivalently elementary quasi-Hopf algebras of finite representation type. The obvious motivation for this is two-fold. On the one hand, the algebras of finite representation type are very important in the representation theory of associative algebras. The study of such algebras has been a central theme in the area all along. Given an interesting class of algebras, one is always tempted to classify those of finite representation type. On the other hand, their associated representation categories are the finite tensor categories in which there are only finitely 
many non-isomorphic indecomposable objects. Such finite tensor categories are simplest after the semisimple ones. It is natural to pay prior attention to these finite tensor categories with very good finiteness property.

The paper is organized as follows. In Section 2 we recall the quiver setting of quasi-quantum groups. Section 3 is devoted to the classification of finitedimensional pointed Majid algebras of corepresentation type. In Section 4 we investigate finite tensor categories by making use of quiver representation theory.

Throughout, we work over the field $\mathbb{C}$ of complex numbers for simplicity. For the convenience of the exposition, we deal mainly with pointed Majid algebras and mention briefly the situation of elementary quasi-Hopf algebras. About general background knowledge, the reader is referred to $[1,2]$ for quivers and representation theory of algebras, to [32, 35, 37] for quasi-quantum groups, and to $[3,5]$ for tensor categories.

\section{Hopf Quivers and Quasi-Quantum Groups}

In this section we recall the quiver framework of quasi-quantum groups for the convenience of the reader.

2.1. Hopf Quivers. A quiver is a quadruple $Q=\left(Q_{0}, Q_{1}, s, t\right)$, where $Q_{0}$ is the set of vertices, $Q_{1}$ is the set of arrows, and $s, t: Q_{1} \longrightarrow Q_{0}$ are two maps assigning respectively the source and the target for each arrow. A path of length $l \geq 1$ in the quiver $Q$ is a finitely ordered sequence of $l$ arrows $a_{l} \cdots a_{1}$ such that $s\left(a_{i+1}\right)=t\left(a_{i}\right)$ for $1 \leq i \leq l-1$. By convention a vertex is said to be a trivial path of length 0 .

For a quiver $Q$, the associated path coalgebra $\mathbb{C} Q$ is the $\mathbb{C}$-space spanned by the set of paths with counit and comultiplication maps defined by $\varepsilon(g)=$ $1, \Delta(g)=g \otimes g$ for each $g \in Q_{0}$, and for each nontrivial path $p=$ $a_{n} \cdots a_{1}, \varepsilon(p)=0$,

$$
\Delta\left(a_{n} \cdots a_{1}\right)=p \otimes s\left(a_{1}\right)+\sum_{i=1}^{n-1} a_{n} \cdots a_{i+1} \otimes a_{i} \cdots a_{1}+t\left(a_{n}\right) \otimes p .
$$

The length of paths gives a natural gradation to the path coalgebra. Let $Q_{n}$ denote the set of paths of length $n$ in $Q$, then $\mathbb{C} Q=\oplus_{n \geq 0} \mathbb{C} Q_{n}$ and $\Delta\left(\mathbb{C} Q_{n}\right) \subseteq \oplus_{n=i+j} \mathbb{C} Q_{i} \otimes \mathbb{C} Q_{j}$. Clearly $\mathbb{C} Q$ is pointed with the set of grouplikes $G(\mathbb{C} Q)=Q_{0}$, and has the following coradical filtration

$$
\mathbb{C} Q_{0} \subseteq \mathbb{C} Q_{0} \oplus \mathbb{C} Q_{1} \subseteq \mathbb{C} Q_{0} \oplus \mathbb{C} Q_{1} \oplus \mathbb{C} Q_{2} \subseteq \cdots
$$


Hence $\mathbb{C} Q$ is coradically graded. The path coalgebras can be presented as cotensor coalgebras, so they are cofree in the category of pointed coalgebras and enjoy a universal mapping property.

According to [11], a quiver $Q$ is said to be a Hopf quiver if the corresponding path coalgebra $\mathbb{C} Q$ admits a graded Hopf algebra structure. Hopf quivers can be determined by ramification data of groups. Let $G$ be a group and denote its set of conjugacy classes by $\mathcal{C}$. A ramification datum $R$ of the group $G$ is a formal sum $\sum_{C \in \mathcal{C}} R_{C} C$ of conjugacy classes with coefficients in $\mathbb{N}=\{0,1,2, \cdots\}$. The corresponding Hopf quiver $Q=Q(G, R)$ is defined as follows: the set of vertices $Q_{0}$ is $G$, and for each $x \in G$ and $c \in C$, there are $R_{C}$ arrows going from $x$ to $c x$. It is clear by definition that $Q(G, R)$ is connected if and only if the the set $\left\{c \in C \mid C \in \mathcal{C}\right.$ with $\left.R_{C} \neq 0\right\}$ generates the group $G$. For a given Hopf quiver $Q$, the set of graded Hopf structures on $\mathbb{C} Q$ is in one-to-one correspondence with the set of $\mathbb{C} Q_{0}$-Hopf bimodule structures on $\mathbb{C} Q_{1}$.

2.2. Quasi-Quantum Groups. We recall explicitly the definitions about Majid algebras only. Those about quasi-Hopf algebras can be written out in a dual manner.

A dual quasi-bialgebra, or Majid bialgebra for short, is a coalgebra $(H, \Delta, \varepsilon)$ equipped with a compatible quasi-algebra structure. Namely, there exist two coalgebra homomorphisms

$$
\mathrm{M}: H \otimes H \longrightarrow H, a \otimes b \mapsto a b, \quad \mu: k \longrightarrow H, \lambda \mapsto \lambda 1_{H}
$$

and a convolution-invertible map $\Phi: H^{\otimes 3} \longrightarrow k$ called reassociator, such that for all $a, b, c, d \in H$ the following equalities hold:

$$
\begin{gathered}
a_{1}\left(b_{1} c_{1}\right) \Phi\left(a_{2}, b_{2}, c_{2}\right)=\Phi\left(a_{1}, b_{1}, c_{1}\right)\left(a_{2} b_{2}\right) c_{2}, \\
1_{H} a=a=a 1_{H}, \\
\Phi\left(a_{1}, b_{1}, c_{1} d_{1}\right) \Phi\left(a_{2} b_{2}, c_{2}, d_{2}\right) \\
=\Phi\left(b_{1}, c_{1}, d_{1}\right) \Phi\left(a_{1}, b_{2} c_{2}, d_{2}\right) \Phi\left(a_{2}, b_{3}, c_{3}\right), \\
\Phi\left(a, 1_{H}, b\right)=\varepsilon(a) \varepsilon(b) .
\end{gathered}
$$

Here and below we use the Sweedler sigma notation $\Delta(a)=a_{1} \otimes a_{2}$ for the coproduct. $H$ is called a Majid algebra if, moreover, there exist a coalgebra antimorphism $\mathcal{S}: H \longrightarrow H$ and two functionals $\alpha, \beta: H \longrightarrow k$ such that 
for all $a \in H$,

$$
\begin{gathered}
\mathcal{S}\left(a_{1}\right) \alpha\left(a_{2}\right) a_{3}=\alpha(a) 1_{H}, \quad a_{1} \beta\left(a_{2}\right) \mathcal{S}\left(a_{3}\right)=\beta(a) 1_{H}, \\
\Phi\left(a_{1}, \mathcal{S}\left(a_{3}\right), a_{5}\right) \beta\left(a_{2}\right) \alpha\left(a_{4}\right)= \\
\Phi^{-1}\left(\mathcal{S}\left(a_{1}\right), a_{3}, \mathcal{S}\left(a_{5}\right)\right) \alpha\left(a_{2}\right) \beta\left(a_{4}\right)=\varepsilon(a) .
\end{gathered}
$$

A Majid algebra $H$ is said to be pointed, if the underlying coalgebra is pointed. For a given pointed Majid algebra $(H, \Delta, \varepsilon, \mathrm{M}, \mu, \Phi, \mathcal{S}, \alpha, \beta)$, let $\left\{H_{n}\right\}_{n \geq 0}$ be its coradical filtration, and gr $H=H_{0} \oplus H_{1} / H_{0} \oplus H_{2} / H_{1} \oplus \cdots$ the corresponding coradically graded coalgebra. Then gr $H$ has an induced graded Majid algebra structure. The corresponding graded reassociator gr $\Phi$ satisfies $\operatorname{gr} \Phi(\bar{a}, \bar{b}, \bar{c})=0$ for all $\bar{a}, \bar{b}, \bar{c} \in \operatorname{gr} H$ unless they all lie in $H_{0}$. Similar condition holds for gr $\alpha$ and $\operatorname{gr} \beta$. In particular, $H_{0}$ is a sub Majid algebra and turns out to be the group algebra $k G$ of the group $G=G(H)$, the set of group-like elements of $H$.

2.3. Quiver Setting for Quasi-Quantum Groups. It is shown in [28] that the path coalgebra $\mathbb{C} Q$ admits a graded Majid algebra structure if and only if the quiver $Q$ is a Hopf quiver. Moreover, for a given Hopf quiver $Q=Q(G, R)$, if we fix a Majid algebra structure on $\mathbb{C} Q_{0}=(\mathbb{C} G, \Phi)$ with quasi-antipode $(\mathcal{S}, \alpha, \beta)$, then the set of graded Majid algebra structures on $\mathbb{C} Q$ with $\mathbb{C} Q_{0}=(\mathbb{C} G, \Phi, \mathcal{S}, \alpha, \beta)$ is in one-to-one correspondence with the set of $(\mathbb{C} G, \Phi)$-Majid bimodule structures on $\mathbb{C} Q_{1}$. According to [13], by transforming the quasi-antipode $(\mathcal{S}, \alpha, \beta)$ via convolution invertible functionals in $\operatorname{Hom}_{\mathbb{C}}(\mathbb{C} G, \mathbb{C})$, one obtains all the graded Majid algebra structures on $\mathbb{C} Q$ with $\mathbb{C} Q_{0}=(\mathbb{C} G, \Phi)$ and an arbitrary quasi-antipode.

The category of Majid bimodules over a general group with an arbitrary 3 -cocycle is characterized in [29] by the admissible collections of projective representations. Let $G$ be a group and $\Phi$ a 3 -cocycle on $G$. Denote by $\mathcal{C}$ the set of conjugacy classes of $G$ and by $Z_{C}$ the centralizer of one of the elements, say $g_{C}$, in the class $C \in \mathcal{C}$. Let $\tilde{\Phi}_{C}$ be a 2-cocycle on $Z_{C}$ defined by

$$
\tilde{\Phi}_{C}(e, f)=\frac{\Phi\left(e, f, g_{C}\right) \Phi\left(e f, f^{-1}, e^{-1}\right) \Phi\left(e, f g_{C}, f^{-1}\right)}{\Phi\left(e f g_{C}, f^{-1}, e^{-1}\right) \Phi\left(e, f, f^{-1}\right)} .
$$

Then the category of $(\mathbb{C} G, \Phi)$-Majid bimodules is equivalent to the product of categories $\prod_{C \in \mathcal{C}}\left(\mathbb{C} Z_{C}, \tilde{\Phi}_{C}\right)$-rep, where $\left(\mathbb{C} Z_{C}, \tilde{\Phi}_{C}\right)$-rep is the category of projective $\tilde{\Phi}_{C}$-representations, or equivalently the left module category of the twisted group algebra $\mathbb{C}^{\tilde{\Phi}_{C}} Z_{C}$ (see [31]). 
Thanks to the Gabriel type theorem in [28], for an arbitrary pointed Majid algebra $H$, its graded version gr $H$ can be realized uniquely as a large sub Majid algebra of some graded Majid algebra structure on a Hopf quiver. By "large" it is meant the sub Majid algebra contains the set of vertices and arrows of the Hopf quiver. Therefore, in principle all pointed Majid algebras are able to be constructed on Hopf quivers. The classification project can be carried out in two steps. The first step is to classify large sub Majid algebras of those on path coalgebras. This gives a classification of graded pointed Majid algebras. The second step is to perform a suitable deformation process to get general pointed Majid algebras from the graded ones.

2.4. Multiplication Formula for Quiver Majid Algebras. In order to construct graded Majid algebras on Hopf quivers, we need to compute the product of paths. It is shown in [28] that the multiplication formula can be given via quantum shuffle product [36].

Suppose that $Q$ is a Hopf quiver with a necessary $\mathbb{C} Q_{0}$-Majid bimodule structure on $\mathbb{C} Q_{1}$. Let $p \in Q_{l}$ be a path. An $n$-thin split of it is a sequence $\left(p_{1}, \cdots, p_{n}\right)$ of vertices and arrows such that the concatenation $p_{n} \cdots p_{1}$ is exactly $p$. These $n$-thin splits are in one-to-one correspondence with the $n$-sequences of $(n-l) 0$ 's and $l 1$ 's. Denote the set of such sequences by $D_{l}^{n}$. Clearly $\left|D_{l}^{n}\right|=\left(\begin{array}{c}n \\ l\end{array}\right)$. For $d=\left(d_{1}, \cdots, d_{n}\right) \in D_{l}^{n}$, the corresponding $n$-thin split is written as $d p=\left((d p)_{1}, \cdots,(d p)_{n}\right)$, in which $(d p)_{i}$ is a vertex if $d_{i}=0$ and an arrow if $d_{i}=1$. Let $\alpha=a_{m} \cdots a_{1}$ and $\beta=b_{n} \cdots b_{1}$ be paths of length $m$ and $n$ respectively. Let $d \in D_{m}^{m+n}$ and $\bar{d} \in D_{n}^{m+n}$ the complement sequence which is obtained from $d$ by replacing each 0 by 1 and each 1 by 0 . Define an element

$$
(\alpha \cdot \beta)_{d}=\left[(d \alpha)_{m+n} \cdot(\bar{d} \beta)_{m+n}\right] \cdots\left[(d \alpha)_{1} \cdot(\bar{d} \beta)_{1}\right]
$$

in $\mathbb{C} Q_{m+n}$, where $\left[(d \alpha)_{i} \cdot(\bar{d} \beta)_{i}\right]$ is understood as the action of $\mathbb{C} Q_{0}$-Majid bimodule on $\mathbb{C} Q_{1}$ and these terms in different brackets are put together by cotensor product, or equivalently concatenation. In terms of these notations, the formula of the product of $\alpha$ and $\beta$ is given as follows:

$$
\alpha \cdot \beta=\sum_{d \in D_{m}^{m+n}}(\alpha \cdot \beta)_{d} .
$$

We should remark that, for general Majid algebras, the product is not associative. So the order must be concerned for the product of more than two terms. 
Convention 2.1. For an arbitrary path $p$ and an integer $n \geq 3$, let $p^{\vec{n}}$ denote the product $\overbrace{(\cdots(}^{n-2} p \cdot p) \cdots) \cdot p$ calculating from the left side. For consistency, when $n<3$, we still use the notation $p^{\vec{n}}$ although there is no risk of associative problem. Similarly we use the notation $p^{\bar{n}}$ for the product calculating from the right side.

\section{Pointed Majid Algebras of Finite Corepresentation Type}

In this section we give an explicit classification of finite-dimensional graded pointed Majid algebras of finite corepresentation type. We start by fixing the Hopf quivers on which such Majid algebras live. Then we calculate all the possible Majid bimodules for the construction. Finally we provide the classification by making use of quiver techniques.

3.1. Determination of Hopf Quivers. Recall that a finite-dimensional algebra is defined to be of finite representation type if the number of the isomorphism classes of indecomposable finite-dimensional modules is finite. A finite-dimensional coalgebra $C$ is said to be of finite corepresentation type if the dual algebra $C^{*}$ is of finite representation type. Since finite-dimensional coalgebras and finite-dimensional algebras are dual to each other, we apply the known results of algebras to the coalgebra setting without explanation.

It is well-known that the module category of a finite-dimensional elementary algebra, or the comodule category of a finite-dimensional pointed coalgebra, can be visualized as the representation category of the corresponding bound quiver (see [1, 8]). Hence the quiver presentation of algebras or coalgebras can provide important information for their representation or corepresentation type. This is the starting point of our classification.

Lemma 3.1. Let $C \neq \mathbb{C}$ be a finite-dimensional pointed coalgebra and assume that its bound quiver $Q$ is a connected Hopf quiver. Then $C$ is of finite corepresentation type if and only if its bound quiver $Q=Q\left(\mathbb{Z}_{n}, g\right)$ where $\mathbb{Z}_{n}=\left\langle g \mid g^{n}=\epsilon\right\rangle$ for some integer $n \geq 1$.

Proof. " $\Rightarrow$ " We denote the vertex group of the quiver $Q$ by $G$ and assume that $Q=Q(G, R)$ for some ramification datum $R=\sum_{C \in \mathcal{C}} R_{C} C$ of $G$. Note that a Hopf quiver is very symmetric, its shape is completely determined once the arrows with source $\epsilon$, the unit element of $G$, are known. For our Hopf quiver $Q$, we claim that the number of arrows starting from $\epsilon$ is 1 . 
Assume otherwise there are at least two arrows with source $\epsilon$. Then the Hopf quiver $Q$ either contains the Kronecker quiver

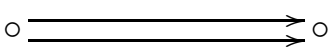

as a sub quiver, or contains a sub quiver of the form

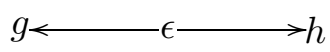

for some $g \neq h$. For the latter case, by the definition of Hopf quivers, in $Q$ there are also arrows as the following

$$
\cdots \longleftarrow h^{-2} g^{2} \longrightarrow h^{-1} g^{2} \longleftarrow h^{-1} g \longrightarrow g .
$$

Since $G$ is a finite group, there is a positive integer $N$ such that $h^{-N+1} g^{N}$ or $h^{-N} g^{N}=\epsilon$. It follows that the quiver $Q$ contains a sub quiver whose underlying graph is a cycle and whose paths are of length less than 2. By the Gabriel type theorem for pointed coalgebras (see e.g. $[7,8]$ ), the bound quiver $Q$ of $C$ contains either of the above two quivers as a sub quiver, hence the corepresentation category of $C$ contains either of their representation categories as a sub category. According to Gabriel's famous classification of quivers of finite representation type [19], both of these two quivers admit infinitely many finite-dimensional indecomposable representations.

On the other hand, since the quiver $Q$ is assumed to be connected, so the number of arrows starting from $\epsilon$ can only be 1 . Assume that $\epsilon \longrightarrow g$ is the unique arrow of $Q$ with source $\epsilon$. By the definition of Hopf quiver, the element $g$ itself must constitute a conjugacy class and generate the group $G$ since $Q$ is connected. Therefore, such a quiver can only be of the following form

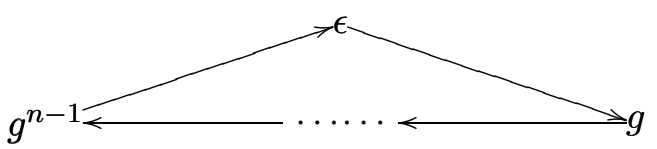

where the set of vertices $\left\{\epsilon, g, \cdots \cdots, g^{n-1}\right\}$ constitutes a cyclic group $\mathbb{Z}_{n}$ of order $n$ and $Q=Q\left(\mathbb{Z}_{n}, g\right)$.

" $\Leftarrow$ " Assume that $Q=Q\left(\mathbb{Z}_{n}, g\right)$ is the bound quiver of $C$, then $C$ is a large sub coalgebra of $\mathbb{C} Q$. Denote $\mathbb{C} Q(d):=\oplus_{i=0}^{d-1} \mathbb{C} Q_{i}$, the $d$-truncated sub coalgebra. Since $C$ is finite-dimensional, $C$ is a sub coalgebra of $\mathbb{C} Q(d)$ for some $d$. It is well-known that $\mathbb{C} Q(d)$ is of finite corepresentation type $[1,2]$ and thus so is $C$.

Assume that $M$ is a finite-dimensional pointed Majid algebra and the corresponding Hopf quiver is $Q$. To avoid the trivial case, we assume that 
$Q$ contains at least one arrow. This excludes the situation for $M$ being cosemisimple. According to the quasi-Hopf analogue of the Cartier-Gabriel decomposition theorem in [28], we may assume without loss of generality that the quiver $Q$ is connected. This is equivalent to saying that the underlying coalgebra of $M$ is connected.

Corollary 3.2. Keep the previous assumption. If $M$ is of finite corepresentation type then the set of group-likes $G(M)=\mathbb{Z}_{n}$ and $Q=Q\left(\mathbb{Z}_{n}, g\right)$ for some integer $n \geq 2$.

Proof. By Lemma 3.1, $Q=Q\left(\mathbb{Z}_{n}, g\right)$ for some $n \geq 1$. We claim the case $n=1$ would not occur. Otherwise, the quiver $Q$ consists of one vertex and one loop. By the multiplication formula (2.8), it is easy to see that the sub Majid algebra generated by the loop is the shuffle algebra in one variable (see e.g. [32]) which is of course infinite-dimensional. By the Gabriel type theorem, this must be contained in gr $M$. This implies that gr $M$, hence $M$, is infinite-dimensional. Contradiction.

3.2. Twisted Group Algebras. From now on we let $Z^{n}$ denote the Hopf quiver $Q\left(\mathbb{Z}_{n}, g\right)$, which is known as the basic cycle of length $n$. In order to classify graded Majid algebras on $Z^{n}$ we need to classify $\left(\mathbb{C Z}_{n}, \Phi\right)$-Majid bimodule structures on $\mathbb{C} Z_{1}^{n}$ for an arbitrary 3-cocycle $\Phi$ on $\mathbb{Z}_{n}$, which can be reduced to the classification of one-dimensional modules over some twisted group algebra $\mathbb{C}^{\sigma} \mathbb{Z}_{n}$ by $[29]$.

Firstly we recall the nontrivial 3 -cocycles on $\mathbb{Z}_{n}$. It is well-known that $H^{3}\left(\mathbb{Z}_{n}, \mathbb{C}^{*}\right) \cong \mathbb{Z}_{n}$, so there are $n$ mutually non-cohomologous 3 -cocycles. We give a list after Lemmas 3.3 and 3.4 of [22]. Let $q$ be a primitive root of unity of order $n$. For any integer $i \in \mathbb{N}$, we denote by $i^{\prime}$ the remainder of division of $i$ by $n$. A list of 3-cocycles on $\mathbb{Z}_{n}$ are

$$
\Phi_{s}\left(g^{i}, g^{j}, g^{k}\right)=\mathbb{q}^{s i\left(j+k-(j+k)^{\prime}\right) / n}
$$

for all $0 \leq s \leq n-1$ and $0 \leq i, j, k \leq n-1$. Obviously, $\Phi_{s}$ is trivial (i.e., cohomologous to a 3-coboundary) if and only if $s=0$.

For a 3 -cocycle $\Phi_{s}$, we define a 2-cocycle $\sigma_{s}$ on $\mathbb{Z}_{n}$ by (2.7) as follows:

$$
\sigma_{s}\left(g^{i}, g^{j}\right):=\frac{\Phi_{s}\left(g^{i}, g^{j}, g\right) \Phi_{s}\left(g^{i+j}, g^{-j}, g^{-i}\right) \Phi_{s}\left(g^{i}, g^{j+1}, g^{-j}\right)}{\Phi_{s}\left(g^{i+j+1}, g^{-j}, g^{-i}\right) \Phi_{s}\left(g^{i}, g^{j}, g^{-j}\right)}
$$

for all $0 \leq i, j \leq n-1$. Consider the associated twisted group algebra $\mathbb{C}^{\sigma_{s}} \mathbb{Z}_{n}$. We denote the multiplication in $\mathbb{C}^{\sigma_{s}} \mathbb{Z}_{n}$ by "*". Thus

$$
g * g=\sigma_{s}(g, g) g^{2}=\frac{\Phi_{s}(g, g, g) \Phi_{s}\left(g^{2}, g^{n-1}, g^{n-1}\right) \Phi_{s}\left(g, g^{2}, g^{n-1}\right)}{\Phi_{s}\left(g^{3}, g^{n-1}, g^{n-1}\right) \Phi_{s}\left(g, g, g^{n-1}\right)} g^{2}=\mathbb{q}^{-s} g^{2} .
$$


Denote $\overbrace{g * \cdots * g}^{i}$ by $g^{* i}$, then we have

Lemma 3.3. In $\mathbb{C}^{\sigma_{s}} \mathbb{Z}_{n}$, we have

$$
g^{i}=\mathbb{q}^{(i-1) s} g^{* i}
$$

for $1 \leq i \leq n$.

Proof. Induction on $i$,

$$
\begin{aligned}
g * g^{i} & =\mathbb{q}^{(i-1) s} g^{*(i+1)}=\sigma_{s}\left(g, g^{i}\right) g^{i+1} \\
& =\frac{\Phi_{s}\left(g, g^{i}, g\right) \Phi_{s}\left(g^{i+1}, g^{n-i}, g^{n-1}\right) \Phi_{s}\left(g, g^{i+1}, g^{n-i}\right)}{\Phi_{s}\left(g^{i+2}, g^{n-i}, g^{n-1}\right) \Phi_{s}\left(g, g^{i}, g^{n-i}\right)} g^{i+1} \\
& =\mathbb{q}^{-s} g^{i+1}
\end{aligned}
$$

This implies that $g^{i+1}=\mathbb{q}^{i s} g^{*(i+1)}$.

Now we consider one-dimensional modules of $\mathbb{C}^{\sigma_{s}} \mathbb{Z}_{n}$. Let the one-dimensional space $V=\mathbb{C} X$ be a $\mathbb{C}^{\sigma_{s}} \mathbb{Z}_{n}$-module with action given by

$$
g \triangleright X=\lambda X
$$

for some $\lambda \in \mathbb{C}$. Then we have

Lemma 3.4. $\lambda^{n}=q^{s}$.

Proof. Indeed, $\lambda^{n} X=g^{* n} \triangleright X=\mathbb{q}^{-(n-1) s} g^{n} \triangleright X=\mathbb{q}^{s} X$.

So $\lambda=q^{s}$ for some $q$ which is an $n$-th root of $\mathbb{q}$. Thus $q$ is a primitive root of unity of order $n^{2}$ when $s \neq 0$. Therefore when $s \neq 0$, the set of one-dimensional $\mathbb{C}^{\sigma_{s}} \mathbb{Z}_{n}$-modules is in one-to-one correspondence with the set of $n$-th roots of $\mathbb{q}$. When $s=0$, we have $\mathbb{C}^{\sigma_{s}} \mathbb{Z}_{n}=\mathbb{C Z}_{n}$ and the the set of one-dimensional $\mathbb{Z}_{n}$-module is in one-to-one correspondence with the set of $n$-th roots of unity.

3.3. Computation of Majid Bimodules. Here and below, let $X_{i}$ denote the arrow $g^{i-1} \longrightarrow g^{i}$ of the Hopf quiver $Z^{n}$ for $1 \leq i \leq n$. For convenience the subscript of $X_{i}$ is read modulo $n$ in some circumstances. By Theorem 3.3 of $[29]$, the $\left(\mathbb{C Z}_{n}, \Phi_{s}\right)$-Majid bimodule structures on $\mathbb{C} Z_{1}^{n}$ can be obtained by extending the $\mathbb{C}^{\sigma_{s}} \mathbb{Z}_{n}$-module structures on the one-dimensional space $\mathbb{C} X_{1}$.

Recall that, for an arbitrary group $G$ and a 3 -cocycle $\Phi$, a $(k G, \Phi)$-Majid bimodule $M$ is simultaneously a $k G$-bicomodule and a quasi $k G$-bimodule such that the quasi-module structure maps are $k G$-bicomodule morphisms. 
Assume that $M=\bigoplus_{g, h \in G}{ }^{g} M^{h}$ is the decomposition into isotypic components, where

$$
{ }^{g} M^{h}=\left\{m \in M \mid \delta_{L}(m)=g \otimes m, \delta_{R}(m)=m \otimes h\right\} .
$$

Here we use $\left(\delta_{L}, \delta_{R}\right)$ to denote the bicomodule structure maps. The quasiactions satisfy the quasi-associativity, namely

$$
\begin{aligned}
& e .(f . m)=\frac{\Phi(e, f, g)}{\Phi(e, f, h)}(e f) . m, \\
& (m . e) . f=\frac{\Phi(h, e, f)}{\Phi(g, e, f)} m .(e f), \\
& (e . m) . f=\frac{\Phi(e, h, f)}{\Phi(e, g, f)} e .(m . f),
\end{aligned}
$$

for all $e, f, g, h \in G$ and $m \in{ }^{g} M^{h}$. These equalities will be used freely.

A $(k G, \Phi)$-Majid bimodule can be associated to an admissible collection of projective modules as follows. We still let $\epsilon$ denote the unit element, and let $\mathcal{C}$ denote the set of conjugacy classes of the group $G$. For each $C \in \mathcal{C}$, let $Z_{C}$ denote the centralizer of one element in $C$, say $g(C)$, and $\tilde{\Phi}_{C}$ the corresponding 2-cocycle $\tilde{\Phi}_{g(C)}$ on $\mathbb{Z}_{C}$ as defined in (2.7), and $M_{C}={ }^{g(C)} M^{\epsilon}$ the $\tilde{\Phi}_{C}$-representation of $Z_{C}$ given by

$$
h \triangleright m=(h . m) . h^{-1}, \quad \forall h \in Z_{C}, m \in M_{C} .
$$

Then $\left(M_{C}\right)_{C \in \mathcal{C}}$ is called the corresponding admissible collections of projective representations of $M$. Conversely, given an admissible collection of projective representations, one can extend it by a twisted version of induced representation to a Majid bimodule, see [29] for detail. This provides the category equivalence mentioned previously in Subsection 2.3.

Now let's get back to our situation of the cyclic group $\mathbb{Z}_{n}$. Let $q$ be an $n$-th root of $\mathbb{q}$ and fix an $\mathbb{C}^{\sigma_{s}} \mathbb{Z}_{n}$-action on $\mathbb{C} X_{1}$ by

$$
g \triangleright X_{1}=q^{s} X_{1}
$$

We extend this $\mathbb{C}^{\sigma_{s}} \mathbb{Z}_{n}$-module to an $\left(\mathbb{C Z}_{n}, \Phi_{s}\right)$-Majid bimodule on $\mathbb{C} Z_{1}^{n}$. The bicomodule structure is defined according to the quiver structure, namely, for $1 \leq i \leq n$,

$$
\delta_{L}\left(X_{i}\right)=g^{i} \otimes X_{i}, \quad \delta_{R}\left(X_{i}\right)=X_{i} \otimes g^{i-1} .
$$

For the quasi bimodule, there is no harm to assume that $g . X_{i}=X_{i+1}$ for $1 \leq i \leq n-1$. With this, we have 
Lemma 3.5. The following equations

$$
\begin{aligned}
g \cdot X_{i} & =X_{i+1} \quad(1 \leq i \leq n-1) \\
g \cdot X_{n} & =\mathbb{q}^{s} X_{1} \\
X_{i} \cdot g & =\mathbb{q}^{-s} q^{-s} X_{i+1} \quad(1 \leq i \leq n)
\end{aligned}
$$

define a quasi $\mathbb{C Z}_{n}$-bimodule on $\mathbb{C} Z_{1}^{n}$ and make it a $\left(\mathbb{C Z}_{n}, \Phi_{s}\right)$-Majid bimodule together with the bicomodule structure defined by (3.9).

Proof. Inductively, we have

$$
X_{i}=g^{i-1} \cdot X_{1}, \quad \text { for } 1 \leq i \leq n .
$$

Thus

$$
g \cdot X_{n}=g \cdot\left(g^{n-1} \cdot X_{1}\right)=\frac{\Phi_{s}\left(g, g^{n-1}, g\right)}{\Phi_{s}\left(g, g^{n-1}, \epsilon\right)} g^{n} \cdot X_{1}=\mathbb{q}^{s} X_{1} .
$$

We proceed to determine the right quasi-action. On the one hand,

$$
g^{n-1} \triangleright X_{1}=\mathbb{q}^{(n-2) s} g^{* n-1} \triangleright X_{1}=\mathbb{q}^{-2 s} q^{(n-1) s} X_{1}=\mathbb{q}^{-s} q^{-s} X_{1} .
$$

On the other hand, by the relation between " " and the quasi-actions "." given by (3.7), we have

$$
g^{n-1} \triangleright X_{1}=\left(g^{n-1} \cdot X_{1}\right) \cdot g=X_{n} \cdot g
$$

Thus $X_{n} \cdot g=\mathbb{q}^{-s} q^{-s} X_{1}$.

Now assume that $X_{n-1} . g=c X_{n}$ for some $c \in \mathbb{C}$. Thus

$$
X_{n} \cdot g=\left(g \cdot X_{n-1}\right) \cdot g=\frac{\Phi_{s}\left(g, g^{n-2}, g\right)}{\Phi_{s}\left(g, g^{n-1}, g\right)} g \cdot\left(X_{n-1} \cdot g\right)=c q^{-s} g \cdot X_{n}=c X_{1}
$$

and $X_{n} . g=\mathbb{q}^{-s} q^{-s} X_{1}$. Therefore, $c=\mathbb{q}^{-s} q^{-s}$. Inductively, assume that $X_{i} . g=\mathbb{q}^{-s} q^{-s} X_{i+1}$ for some $i \leq n-1$. Suppose that $X_{i-1} . g=c X_{i}$ for some $c \in \mathbb{C}$, then

$$
\mathbb{q}^{-s} q^{-s} X_{i+1}=X_{i} \cdot g=\left(g \cdot X_{i-1}\right) \cdot g=g \cdot\left(X_{i-1} \cdot g\right)=c g \cdot X_{i}=c X_{i+1} .
$$

Thus $c=\mathbb{q}^{-s} q^{-s}$.

It is straightforward to verify that the quasi-bimodule structure maps are bicomodule morphisms. This completes the proof. 
3.4. Graded Majid Algebras on $Z^{n}$. In this subsection we calculate the graded Majid algebra on the quiver $Z^{n}$ associated to the $\left(\mathbb{C Z}_{n}, \Phi\right)$-Majid bimodule given in Lemma 3.5.

Firstly we need to fix some notations. For any $\hbar \in \mathbb{C}$, define $l_{\hbar}=1+\hbar+$ $\cdots+\hbar^{l-1}$ and $l !_{\hbar}=1_{\hbar} \cdots l_{\hbar}$. The Gaussian binomial coefficient is defined by $\left(\begin{array}{c}l+m \\ l\end{array}\right)_{\hbar}:=\frac{(l+m) !_{\hbar}}{l !_{\hbar} m !_{\hbar}}$.

In the path coalgebra $\mathbb{C} Z^{n}$, let $p_{i}^{l}$ denote the path starting from $g^{i}$ with length $l$. The index " $i$ " is read modulo $n$ when there is no risk of confusion. We keep the $\left(\mathbb{C} \mathbb{Z}_{n}, \Phi_{s}\right)$-Majid bimodule on $\mathbb{C} Z_{1}^{n}$ as in Lemma 3.5 and consider the associated graded Majid algebra as given in [28]. Let $\mathbb{C} Z^{n}(s, q)$ denote the resulted Majid algebra on the path coalgebra $\mathbb{C} Z Z^{n}$ and "." denote its multiplication. Note that the quasi-antipode $(\mathcal{S}, \alpha, \beta)$ of $\mathbb{C} Z^{n}(s, q)$ satisfies $\mathcal{S}(g)=g^{-1}, \alpha(g)=1, \beta(g)=1 / \Phi_{s}\left(g, g^{-1}, g\right)$ for all $g \in \mathbb{Z}_{n}$.

Lemma 3.6. For any natural number $l$, we have

$$
X_{1}^{\vec{l}}=l !_{\mathbb{Q}^{-s} q^{-s}} p_{0}^{l}, \quad X_{1}^{\overleftarrow{l}}=\mathbb{q}^{s l^{\prime}\left(l-l^{\prime}\right) / n} l !_{\mathbb{q}^{-s} q^{-s}} p_{0}^{l}
$$

Proof. By induction on $l$. Firstly, we have

$$
\begin{aligned}
X_{1} \cdot X_{1} & =\left[g \cdot X_{1}\right]\left[X_{1} \cdot \epsilon\right]+\left[X_{1} \cdot g\right]\left[\epsilon \cdot X_{1}\right] \\
& =\left(1+\mathbb{q}^{-s} q^{-s}\right) X_{2} X_{1}=2_{\mathbb{q}^{-s} q^{-s}} X_{2} X_{1} .
\end{aligned}
$$

Assume $l=a n+i$ with $0 \leq i \leq n-1$ and $X_{1}^{l-1}=(l-1) !_{\mathbb{q}^{-s}} q^{-s} p_{0}^{l-1}$, then

$$
\begin{aligned}
X_{1}^{\vec{l}}= & X_{1}^{l-1} \cdot X_{1}=(l-1) !_{\mathbb{Q}^{-s}} q^{-s} p_{0}^{l-1} \cdot X_{1} \\
= & (l-1) !_{\mathbb{q}^{-s} q^{-s}}\left(\left[g^{i} \cdot X_{1}\right]\left[X_{i} \cdot \epsilon\right] \cdots\left[X_{1} \cdot \epsilon\right]\right. \\
& \left.+\cdots+\left[X_{i} \cdot g\right] \cdots\left[X_{1} \cdot g\right]\left[\epsilon \cdot X_{1}\right]\right) \\
= & (l-1) !_{\mathbb{q}^{-s} q^{-s}}\left(1+\mathbb{q}^{-s} q^{-s}+\cdots+\mathbb{q}^{-(l-1) s} q^{-(l-1) s}\right) p_{0}^{l} \\
= & l !_{\mathbb{q}^{-s} q^{-s}} p_{0}^{l} .
\end{aligned}
$$

Similarly, for $l=a n+i$ as above we have

$$
\begin{aligned}
X_{1}^{l+1}= & X_{1} \cdot X_{1}{ }_{1}^{l}=\mathbb{q}^{s i a} l !_{\mathbb{q}^{-s} q^{-s}} X_{1} \cdot p_{0}^{l} \\
= & \mathbb{q}^{s i a} l !_{\mathbb{q}^{-s} q^{-s}}\left(\left(\mathbb{q}^{-s} q^{-s}\right)^{i}+\cdots\left(\mathbb{q}^{-s} q^{-s}\right)^{1}+1\right. \\
& \left.+\mathbb{q}^{s} n_{\mathbb{q}^{-s} q^{-s}}+\cdots+\mathbb{q}^{s a} n_{\mathbb{q}^{-s}} q^{-s}\right) p_{0}^{l+1} \\
= & \mathbb{q}^{s i a} l !_{\mathbb{q}^{-s} q^{-s}} \mathbb{q}^{s a}(l+1)_{\mathbb{q}^{-s} q^{-s}} p_{0}^{l+1} \\
= & \mathbb{q}^{s(i+1) a}(l+1) !_{\mathbb{Q}^{-s} q^{-s}} p_{0}^{l+1} .
\end{aligned}
$$


Lemma 3.7. For all $0 \leq i, j \leq n-1$ and all non-negative integers $a, b$,

$$
X_{1}^{a \overrightarrow{n+i}} \cdot X_{1}^{b n+j}= \begin{cases}\mathbb{q}^{-(a+1)(i+j) s} X_{1}^{(a+b) \grave{n+(i+j)}} & \text { if } i+j>n-1 \\ \mathbb{q}^{-a(i+j) s} X_{1}^{(a+b) n+(i+j)} & \text { if } i+j \leq n-1 .\end{cases}
$$

Proof. Consider the multiplication of $X_{1}^{a n+i-1}, X_{1}$ and $X_{1}^{b n+j}$. By the quasiassociativity axiom (2.1) of Majid algebras, we have the following equality

$$
X_{1}^{a \overrightarrow{n+i}} \cdot X_{1}^{b n+j}=\frac{\Phi(\epsilon, \epsilon, \epsilon)}{\Phi\left(g^{i-1}, g, g^{j}\right)} X_{1}^{a n+\overrightarrow{+i-1}} \cdot X_{1}^{b n+j+1}
$$

By this, it follows that the coefficient is trivial unless $j=n-1$. Thus if $i+j>n-1$, by making use of the previous equation iteratively we have

$$
\begin{aligned}
& X_{1}^{a \overrightarrow{n+i}} \cdot X_{1}^{b n+j}=X_{1}^{a n+(i+\vec{j}+1-n)} \cdot X_{1}^{b n+\check{L}-1} \\
& =\mathbb{q}^{-(i+j) s} X_{1}^{a n+(\overrightarrow{i+j-n)}} \cdot X_{1}^{(b+\overleftarrow{1}) n} \\
& =\mathbb{q}^{-(i+j) s} X_{1}^{(a-\overrightarrow{1}) n+i} \cdot X_{1}^{(b+\overleftarrow{1)} n+j} \\
& =\ldots \\
& =\mathbb{q}^{-a(i+j) s} X_{1}^{\vec{i}} \cdot X_{1}^{(a+\overleftarrow{b} n+j} \\
& =\mathbb{q}^{-a(i+j) s} X_{1}^{i+j+1-n} \cdot X_{1}^{(a+b) \stackrel{\check{n}+n-1}{ }} \\
& =\mathbb{q}^{(-a-1)(i+j) s} X_{1}^{i+\vec{j}-n} \cdot X_{1}^{(a+\check{b+1}) n} \\
& =\mathbb{q}^{-(a+1)(i+j) s} X_{1}^{(a+b) n+(i+j)} \text {. }
\end{aligned}
$$

The case of $i+j \leq n-1$ can be proved in the same manner.

With these preparations, now we can give the product formula.

Proposition 3.8. For all non-negative integers $l, m$, we have

$$
p_{0}^{l} \cdot p_{0}^{m}=\mathbb{q}^{s l^{\prime}\left(m-m^{\prime}\right) / n}\left(\begin{array}{c}
l+m \\
l
\end{array}\right)_{\mathbb{q}^{-s} q^{-s}} p_{0}^{l+m}
$$

Proof. Assume that $l=a n+i, m=b n+j$ with $0 \leq i, j \leq n-1$. We only prove the formula when $i+j \leq n-1$ since the case $i+j>n-1$ can be 
proved similarly. Indeed, by Lemmas 3.6 and 3.7, we have

$$
\begin{aligned}
p_{0}^{l} \cdot p_{0}^{m} & =\frac{1}{l !_{\mathbb{Q}^{-s} q^{-s}}} \frac{1}{\mathbb{q}^{s j b} m !_{\mathbb{q}^{-s} q^{-s}}} X_{1}^{\vec{l}} \cdot X_{1}^{\bar{m}} \\
& =\frac{\mathbb{q}^{-s j b} \mathbb{Q}^{-a(i+j) s}}{l !_{\mathbb{Q}^{-s} q^{-s}} m !_{\mathbb{q}^{-s} q^{-s}}} X_{1}^{l+m} \\
& =\frac{\mathbb{q}^{-s j b} \mathbb{q}^{-a(i+j) s}}{l !_{\mathbb{Q}^{-s} q^{-s}} m !_{\mathbb{q}^{-s} q^{-s}}} \mathbb{q}^{s(i+j)(a+b)}(l+m) !_{\mathbb{q}^{-s} q^{-s}} p_{0}^{l+m} \\
& =\mathbb{q}^{s i b}\left(\begin{array}{c}
l+m \\
l
\end{array}\right)_{\mathbb{q}^{-s} q^{-s}} p_{0}^{l+m} .
\end{aligned}
$$

Observe that

$$
g^{i} \cdot p_{0}^{l}=\mathbb{q}^{s i\left(l-l^{\prime}\right) / n} p_{i}^{l}, \quad p_{0}^{l} \cdot g^{i}=\mathbb{q}^{-s i l} q^{-s i l} p_{i}^{l} .
$$

Then the previous product formula can be extended to general paths as follows. We leave the proof to the interested reader.

Corollary 3.9. For all $0 \leq i, j \leq n-1$ and all non-negative integers $l, m$, we have in $\mathbb{C} Z^{n}(s, q)$ the following multiplication formula

$$
p_{i}^{l} \cdot p_{j}^{m}=\mathbb{q}^{-s j l} q^{-s j l} \mathbb{q}^{s\left(i+l^{\prime}\right)\left[m+j-(m+j)^{\prime}\right] / n}\left(\begin{array}{c}
l+m \\
l
\end{array}\right)_{\mathbb{q}^{-s} q^{-s}} p_{i+j}^{l+m} .
$$

We remark that the set $\left\{\mathbb{C} Z^{n}(0, q) \mid q^{n}=1\right\} \cup\left\{\mathbb{C} Z^{n}(s, q) \mid 1 \leq s \leq n-\right.$ $1, q$ is a primitive root of unity of order $\left.n^{2}\right\}$ gives an explicit classification of graded Majid algebras on the path coalgebra $\mathbb{C} Z^{n}$ by neglecting minor difference of the quasi-antipodes [13]. In particular, when $s=0$, the set $\left\{\mathbb{C} Z^{n}(0, q) \mid q^{n}=1\right\}$ gives a classification of graded Hopf algebras on $\mathbb{C} Z^{n}$, which recovers the result in $[9]$; when $s \neq 0$, the set $\left\{\mathbb{C} Z^{n}(s, q) \mid 1 \leq s \leq\right.$ $n-1, q$ is a primitive root of unity of order $\left.n^{2}\right\}$ gives a classification of nontrivial graded Majid algebras (i.e., not gauge equivalent to Hopf algebras) on $\mathbb{C} Z^{n}$ by $[29]$.

3.5. Finite Sub Majid Algebra on $Z^{n}$. In this subsection we investigate the possible finite-dimensional graded large sub Majid algebras of $\mathbb{C} Z^{n}(s, q)$.

Proposition 3.10. There is a unique finite-dimensional graded large sub Majid algebra of $\mathbb{C} Z^{n}(s, q)$. In addition, such unique Majid algebra is generated by $g$ and $X_{1}$.

Proof. By definition, the smallest graded large sub Majid algebra of $\mathbb{C} Z^{n}(s, q)$ is the one generated by the set of vertices and arrows, which can be given 
by $g$ and $X_{1}$ clearly. Assume that the multiplicative order of $\mathbb{q}^{-s} q^{-s}$ is $d$. Then by Lemmas 3.5 and 3.6, the quasi-algebra generated by $g$ and $X_{1}$ has $\left\{g^{i} \cdot X_{1}^{l} \mid 0 \leq i \leq n-1,0 \leq l \leq d-1\right\}$ as a basis. Thus by (3.13) the set $\left\{p_{i}^{l} \mid 0 \leq i \leq n-1,0 \leq l \leq d-1\right\}$ is also a basis. Clearly the space spanned by these paths is closed under the coproduct of the path coalgebra, and also closed under the counit and the quasi-antipode of $\mathbb{C} Z^{n}(s, q)$. Therefore it is an $n d$-dimensional graded large sub Majid algebra. We denote this graded Majid algebra by $M(n, s, q)$.

It remains to prove that any graded large sub Majid algebra of $\mathbb{C} Z^{n}(s, q)$ is infinite-dimensional if it strictly contains $M(n, s, q)$. Assume that $M$ is such a Majid algebra. Then consider a nontrivial homogeneous space of degree $l \geq d$ in $M$. Note that such a space must be spanned by some paths of length $l$. Since there is only one path of length $l$ with fixed source and target, it follows by the axioms of coalgebras that there are paths of length $l$ lies in $H$. Then by (3.13) all the paths of length $l$ lie in $H$. Consider the coproduct of these paths, it follows that all the paths of length $d$ must lie in $H$. Now by making use of Proposition 3.8 with induction, we have $\left(p_{0}^{d}\right)^{\vec{m}}=m ! p_{0}^{m d}$. It follows that $p_{0}^{m d} \in H$ for all $m \geq 0$. That means $H$ must be infinite-dimensional. We are done.

Now we can conclude that the set $\left\{M(n, 0, q) \mid q^{n}=1\right\} \cup\{M(n, s, q) \mid 1 \leq$ $s \leq n-1, q$ is a primitive root of unity of order $\left.n^{2}\right\}$ provides a complete classification of finite-dimensional graded large sub Majid algebra on the Hopf quiver $Z^{n}$. Note that the set $\left\{M(n, 0, q) \mid q^{n}=1\right\}$ are the usual Hopf algebras, called generalized Taft algebras in [30]. This gives a classification of finite-dimensional graded Hopf algebras on the quiver $Z^{n}$. While the set $\left\{M(n, s, q) \mid 1 \leq s \leq n-1, q\right.$ is a primitive root of unity of order $\left.n^{2}\right\}$ gives a classification of finite-dimensional graded non-trivial Majid algebras on $Z^{n}$.

It is also worthy to remark that the underlying coalgebras of these Majid algebras are truncated subcoalgebras of $\mathbb{C} Z^{n}$, namely they have a basis consisting of all the paths of length smaller than some fixed integer $d \geq 2$. In particular, they are monomial in the sense of [6]. The set $\left\{M(n, 0, q) \mid q^{n}=\right.$ $1\} \cup\left\{M(n, s, q) \mid 1 \leq s \leq n-1, q\right.$ is a primitive root of unity of order $\left.n^{2}\right\}$ also gives a classification of connected monomial graded Majid algebras, which contains the classification result of monomial Hopf algebras in [6].

Corollary 3.11. Let $\mathbb{C} Z^{n}(d)$ denote the truncated sub coalgebra of $\mathbb{C} Z^{n}$ spanned by the paths of length smaller than some fixed integer $d \geq 2$. Then $\mathbb{C} Z^{n}(d)$ admits a graded Majid algebra structure if and only if $d \mid n$, or $d=$ 
$\frac{n^{2}}{\left(s, n^{2}\right)}$ for some $1 \leq s \leq n-1$. In this case, the Majid algebra on $\mathbb{C} Z^{n}(d)$ is gauge equivalent to a Hopf algebra if and only if $d \mid n$.

3.6. Classification Results. Now we are ready to give the main result. In the following we always assume that, for a pointed Majid algebra $M$ with set of group-like elements $G(M)$, its quasi-antipode $(\mathcal{S}, \alpha, \beta)$ satisfies $\mathcal{S}(g)=$ $g^{-1}, \alpha(g)=1, \beta(g)=1 / \Phi\left(g, g^{-1}, g\right)$ for all $g \in G(M)$. The observation of Drinfeld [13] guarantees that this assumption is harmless.

Theorem 3.12. Suppose that $M \neq \mathbb{C}$ is a finite-dimensional graded pointed Majid algebra with connected underlying coalgebra. If $M$ is of finite corepresentation type, then $M \cong M(n, s, q)$ for some positive integers $n \geq 2$ and $0 \leq s \leq n-1$, and $q$ is an $n$-th root of unity if $s=0$, or some primitive root of unity of order $n^{2}$ if $s \neq 0$.

Proof. By the assumption, the corresponding Hopf quiver of $M$ is $Z^{n}$ for some $n \geq 2$ according to Corollary 3.2. Now by the Gabriel type theorem for Majid algebra and Subsection 3.4, $M$ can be viewed as a graded large sub Majid algebra of some $\mathbb{C} Z^{n}(s, q)$. By Proposition 3.10, there is only one possible finite-dimensional graded large sub Majid algebra $M(n, s, q)$. So $M$ can only be one of the $M(n, s, q)$.

On the other hand, since the $M(n, s, q)$ are truncated sub coalgebra of $\mathbb{C Z}^{n}$, so they are of finite corepresentation type as mentioned in Lemma 3.1. This completes the proof.

With a help of this theorem, in the following we deal with not necessarily graded situation by making use of deformation theory [23] and a deep result from geometric methods of representation theory [21]. Without loss of generality, let $M$ be a finite-dimensional pointed Majid algebra with connected underlying coalgebra, that is, its bound quiver is connected. As before, we exclude the trivial case for $M=\mathbb{C}$.

Corollary 3.13. Keep the above assumption. Then $M$ is of finite corepresentation type if and only if gr $M \cong M(n, s, q)$ for some appropriate $n, s, q$.

Proof. First suppose that $M$ is of finite corepresentation type. Consider its underlying coalgebra and apply the Gabriel type theorem. Since $M$ and gr $M$ share the same quiver, so there is a unique Hopf quiver $Q$ such that $M$ can be viewed as a large sub coalgebra of the path coalgebra $\mathbb{C} Q$. Now Lemma 3.1 applies, that is, when $M$ is of corepresentation type, then the 
quiver $Q$ can only be $Z^{n}$ for some integer $n \geq 2$. According to Proposition 3.10 , the graded version gr $M$ of $M$ is isomorphic to some $M(n, s, q)$.

Conversely, suppose gr $M \cong M(n, s, q)$. Note that $M$ is a deformation of $\operatorname{gr} M$ (it is also said that $\operatorname{gr} M$ is a degeneration of $M$ ). Then that the Majid algebra $M(n, s, q)$ is of finite corepresentation type implies that so is $M$, according to the famous theorem of Gabriel [21] which says that finite representation type is open.

To complete the classification of non-graded connected pointed Majid algebras of finite corepresentation type, it suffices to calculate all the deformations of $M(n, s, q)$. Note that finite-dimensional Majid algebras are coFrobenius (i.e., the dual algebra is Frobenius) according to [27, 4], then by the same argument as in Section 2 of [6] we can conclude that the underlying coalgebra of a connected pointed Majid algebras of finite corepresentation type is isomorphic to a truncated sub coalgebra of $\mathbb{C} Z^{n}$. It follows that one only needs to calculate the coalgebra-preserving deformation of $M(n, s, q)$, which is a quasi analogue of the preferred deformation of Hopf algebras [24].

3.7. Some Remarks. We conclude this section with some remarks.

(1) The preferred deformations for $M(n, 0, q)$ were explicitly given in [6]. For $s \neq 0$, it seems that the preferred deformations for $M(n, s, q)$ are much more complicated since the deformation of reassociators gets involved. We leave this problem for future work.

(2) For not necessarily connected situation, the underlying coalgebra of a finite-dimensional pointed Majid algebras is a direct sum of finite copies of some $\mathbb{C} Z^{n}(d)$ and the Majid algebra is a crossed product of some deformation of $M(n, s, q)$ with a group twisted by a three cocycle [28].

(3) Our classification of finite-dimensional pointed Majid algebras of finite corepresentation type contains the corresponding classification result for pointed Hopf algebras, which was given in [34] by different method.

(4) A standard dualization process gives parallel classification results for elementary quasi-Hopf algebras of finite representation type. Some of the dual of $M(n, s, q)$ appeared in previous works of Etingof and Gelaki [14, 15, $16,22]$.

\section{Tensor CAtegories of Finite tyPE}

As an application, we will classify a class of tensor categories of finite type in this section. In addition, some information of these finite tensor categories are given by making use of quiver representation theory. 
4.1. Finite Tensor Categories. By a tensor category we mean an abelian rigid monoidal category over $\mathbb{C}$ in which the neutral object $\mathbf{1}$ is simple. A tensor category $\mathscr{C}$ is said to be finite if

(1) $\mathscr{C}$ has finitely many simple objects,

(2) any object has finite length, and

(3) any simple object admits a projective cover.

For a finite tensor category $\mathscr{C}$, we denote its Grothendieck ring by $\operatorname{Gr}(\mathscr{C})$. It is a free abelian group of finite rank, whose basis $S$ is the set of isomorphism classes of simple objects in $\mathscr{C}$. Let $X \in \mathscr{C}$, then its Frobenius-Perron dimension $d_{+}(X)$ is defined to be the largest non-negative real eigenvalue of the matrix of left multiplication in the Grothendieck ring by $X$ under the basis $S$. For more knowledge about finite tensor categories, see $[17,18,5]$ and references therein.

The following result of Etingof and Ostrik [18] provides the close relation between finite tensor categories and finite quasi-quantum groups.

Lemma 4.1. (Etingof-Ostrik) For a finite tensor category $\mathscr{C}$, it is tensor equivalent to the representation category Rep $H$ of a finite-dimensional quasi-Hopf algebra $H$ if and only if the Frobenius-Perron dimensions of objects in $\mathscr{C}$ are integers.

Since Majid algebras are dual of quasi-Hopf algebras, so a finite tensor category $\mathscr{C}$ is tensor equivalent to the corepresentation category Corep $M$ of a finite-dimensional Majid algebra $M$ if and only if the Frobenius-Perron dimensions of objects in $\mathscr{C}$ are integers.

4.2. Tensor Categories of Finite Type. The simplest finite tensor categories are of course the semisimple ones. After the semisimple situation, the simplest ones are those having finitely many isomorphism classes of indecomposable objects in view of the Krull-Remak-Schmidt property of finite tensor categories. A finite tensor category $\mathscr{C}$ with this property must be equivalent to the representation category $\operatorname{Rep} A$ of a finite-dimensional algebra $A$ of finite representation type, or the corepresentation category Corep $C$ of a finite-dimensional coalgebra $C$ of finite corepresentation type. Inspired by this, such tensor categories are said to be of finite type.

By Lemma 4.1, the classification of tensor categories of finite type whose objects have integer Frobenius-Perron dimensions is equivalent to the classification of finite-dimensional quasi-Hopf algebras of finite representation type, or finite-dimensional Majid algebras of finite corepresentation type. 
Now the results of Section 3 can be applied to classify some class of such tensor categories.

4.3. Some Classification Results. Let $\mathscr{C}$ be a finite tensor category and assume that $\mathscr{C}=\operatorname{Corep} C$ as an abelian category for a finite-dimensional coalgebra $C$. We say that $\mathscr{C}$ is connected if the coalgebra $C$ is connected, that is, the dual algebra $C^{*}$ of $C$ is indecomposable [2]. To avoid the trivial case, in the following we always assume that $\mathscr{C}$ has at least two simple objects.

Theorem 4.2. Assume that $\mathscr{C}$ is a tensor category of finite type. If every simple object of $\mathscr{C}$ has Frobenius-Perron dimension 1, then as an abelian category $\mathscr{C}$ is equivalent to direct product of finite copies of Corep $\mathbb{C} Z^{n}(d)$ with $d \mid n$, or $d=\frac{n^{2}}{\left(s, n^{2}\right)}$ for some $1 \leq s \leq n-1$.

Proof. By the assumption, first of all we have $\mathscr{C}=$ Corep $C$ as an abelian category for some finite-dimensional pointed coalgebra $C$. By the property of Frobenius-Perron dimension, the fact that every simple object of $\mathscr{C}$ has Frobenius-Perron dimension 1 implies all objects of $\mathscr{C}$ have integer Frobenius-Perron dimensions. Then $C$ must be a pointed Majid algebra of finite corepresentation type by Lemma 4.1. Now the theorem follows immediately from Subsections 3.6 and 3.7.

The corepresentation category Corep $\mathbb{C} Z^{n}(d)$ is well understood, see for example [1, 2] and also [30] for its Auslander-Reiten quiver. In particular, $\mathbb{C} Z^{n}(d)$ is Nakayama $[1,2]$. Therefore, we have

Corollary 4.3. Assume that $\mathscr{C}$ is a tensor category of finite type and that every simple object of $\mathscr{C}$ has Frobenius-Perron dimension 1, then every indecomposable object $X \in \mathscr{C}$ is uniserial, that is, the set of sub objects of $X$ is totally ordered by inclusion.

Combine Corollary 3.13 and Lemma 4.1 we have

Corollary 4.4. Assume that $\mathscr{C}$ is a connected tensor category of finite type. If every simple object of $\mathscr{C}$ has Frobenius-Perron dimension 1, then as a tensor category $\mathscr{C}$ is equivalent to Corep $M$ for some pointed Majid algebras $M$ with $\operatorname{gr} M \cong M(n, s, q)$.

Acknowledgements: The research of the first author was supported by the NSF of China under grant number 10601052. The research of the second author was supported by the NSF of China under grant number 10801069 . 


\section{REFERENCES}

[1] I. Assem, D. Simson, A. Skowronski, Elements of the Representation Theory of Associative Algebras. Vol. 1. Techniques of Representation Theory. London Mathematical Society Student Texts, 65. Cambridge University Press, Cambridge, 2006.

[2] M. Auslander, I. Reiten, S.O. Smalø, Representation Theory of Artin Algebras, Cambridge Studies in Adv. Math. 36, Cambridge Univ. Press, 1995.

[3] B. Bakalov, A. Kirilov Jr., Lectures on tensor categories and modular funtors, AMS, Providence, (2001).

[4] D. Bulacu, S. Caenepeel, Integrals for (dual) quasi-Hopf algebras. Applications, J. Algebra 266(2) (2003) 552-583.

[5] D. Calaque, P. Etingof, Lectures on tensor categories, math.QA/0401246.

[6] X.-W. Chen, H.-L. Huang, Y. Ye, P. Zhang, Monomial Hopf algebras, J. Algebra 275 (2004) 212-232.

[7] X.-W. Chen, H.-L. Huang, P. Zhang, Dual Gabriel theorem with applications, Sci. in China series A Math. 49(1) (2006) 9-26.

[8] W. Chin, A brief introduction to coalgebra representation theory, Lecture Notes in Pure and Appl. Math. vol. 237, Dekker (2004), pp. 109-131.

[9] C. Cibils, A quiver quantum group, Comm. Math. Phys. 157 (1993) 459-477.

[10] C. Cibils, M. Rosso, Algèbres des chemins quantiques, Adv. Math. 125 (1997) 171199.

[11] C. Cibils, M. Rosso, Hopf quivers, J. Algebra 254 (2002) 241-251.

[12] V.G. Drinfeld, Quantum groups, Proceedings of the International Congress of Mathematicians, Vol. 1, 2 (Berkeley, Calif., 1986), 798-820, Amer. Math. Soc., Providence, RI, 1987.

[13] V.G. Drinfeld, Quasi-Hopf algebras, Leningrad Math. J. 1 (1990) 1419-1457.

[14] P. Etingof, S. Gelaki, Finite dimensional quasi-Hopf algebras with radical of codimension 2, Math. Res. Lett. 11 (2004) 685-696.

[15] P. Etingof, S. Gelaki, On radically graded finite-dimensional quasi-Hopf algebras, Mosc. Math. J. 5(2) (2005) 371-378.

[16] P. Etingof, S. Gelaki, Liftings of graded quasi-Hopf algebras with radical of prime codimension, J. Pure Appl. Algebra 205(2) (2006) 310-322.

[17] P. Etingof, D. Nikshych, V. Ostrik, On fusion categories, Ann. of Math. 162 (2005) 581-642.

[18] P. Etingof, V. Ostrik, Finite tensor categories, Moscow Math. J. 4 (2004) 627-654.

[19] P. Gabriel, Unzerlegbare Darstellungen I, Manuscripta Math. 6 (1972) 71-103.

[20] P. Gabriel, Indecomposable representations II, Symposia Mathematica, Vol. XI (Convegno di Algebra Commutativa, INDAM, Rome, 1971), 81-104, Academic Press, London, 1973.

[21] P. Gabriel, Finite representation type is open, in: Representations of Algebras, Lecture Notes in Math., vol. 488, Springer Verlag, 1975, pp. 132-155.

[22] S. Gelaki, Basic quasi-Hopf algebras of dimension $n^{3}$, J. Pure Appl. Algebra 198(1-3) (2005) 165-174.

[23] M. Gerstenhaer, On the deformation of rings and algebras, Ann. of Math. 79(2) (1964) 59-103. 
[24] M. Gerstenhaber, S.D. Schack, Bialgebra cohomology, deformations, and quantum groups, Proc. Nat. Acad. Sci. 87 (1990) 478-481.

[25] E.L. Green, Constructing quantum groups and Hopf algebras from coverings, J. Algebra 176 (1995) 12-33.

[26] E.L. Green, Ø. Solberg, Basic Hopf algebras and quantum groups, Math. Z. 229 (1998) 45-76.

[27] F. Hausser, F. Nill, Integral theory for quasi-Hopf algebras, math.QA/9904164.

[28] H.-L. Huang, Quiver approaches to quasi-Hopf algebras, J. Math. Phys. 50(4) (2009) 043501-1 - 043501-9. arXiv:0902.1620.

[29] H.-L. Huang, From projective representations to quasi-quantum groups, submitted, arXiv:0903.1472.

[30] H.-L. Huang, H.-X. Chen, P. Zhang, Generalized Taft algebras, Algebra Colloq. 11(03) (2004) 313-320.

[31] G. Karpilovsky, Projecive Representations of Finite Groups, Marcel Dekker, New York, 1985.

[32] C. Kassel, Quantum Groups, Graduate Texts in Math. 155, Springer-Verlag, New York, 1995.

[33] G. Liu, Classification of finite dimensional basic Hopf algebras according to their representation type, Contemp. Math. 478 (2009) 103-124.

[34] G. Liu, F. Li, Pointed Hopf algebras of finite corepresentation type and their classifications, Proceedings of AMS, 135(3) (2007) 649-657.

[35] S. Majid, Foundations of Quantum Group Theory, Cambridge University Press, Cambridge, 1995.

[36] M. Rosso, Quantum groups and quantum shuffles, Invent. Math. 133 (1998) 399-416.

[37] S. Shnider, S. Sternberg, Quantum Groups: From Coalgebras to Drinfeld Algebras, International Press Inc., Boston, 1993.

[38] F. Van Oystaeyen, P. Zhang, Quiver Hopf algebras, J. Algebra 280(2) (2004) 577-589.

School of Mathematics, Shandong University, Jinan, Shandong 250100, China E-mail address: hualin@sdu.edu.cn

Department of Mathematics, Nanjing University, Nanjing, Jiangsu 210093, CHINA

E-mail address: gxliu@nju.edu.cn

Department of Mathematics, University of Science and Technology of China, Hefei, Anhui 230026, China

E-mail address: yeyu@ustc.edu.cn 